\title{
Enhancement of the effects of gemcitabine against pancreatic cancer by oridonin via the mitochondrial caspase-dependent signaling pathway
}

\author{
DIAN-LEI LIU ${ }^{1 *}$, HE-QI BU ${ }^{2 *}$, HAI-MIN JIN ${ }^{1}$, JIN-FENG ZHAO ${ }^{1}$, YE LI $^{1}$ and HAI HUANG ${ }^{1}$ \\ ${ }^{1}$ Department of General Surgery, Guangxing Affiliated Hospital of Zhejiang Chinese Medical University, \\ Hangzhou, Zhejiang 310007; ${ }^{2}$ Department of Anorectal Surgery, Tongde Hospital of Zhejiang Province, \\ Hangzhou, Zhejiang 310012, P.R. China
}

Received April 9, 2014; Accepted July 27, 2014

DOI: $10.3892 / \mathrm{mmr} .2014 .2584$

\begin{abstract}
Gemcitabine is a first-line chemotherapeutic agent used in the treatment of pancreatic cancer; however resistance of the disease to the drug often develops over time. Agents that can either enhance the effects of gemcitabine, or help to overcome the chemoresistance to the drug are needed for the successful treatment of pancreatic cancer. Oridonin is one such agent which is safe and multi-targeted and has previously been shown to induce apoptosis in other tumor cells, through mitochondrial signaling pathways. The aims of the present study were to evaluate whether oridonin may enhance the effects of gemcitabine on pancreatic cancer in vitro and to investigate the possible mechanisms of this enhancement. In vitro studies have previously shown that oridonin can inhibit the proliferation of the PANC-1 pancreatic cancer cell line, and potentiate gemcitabine-induced apoptosis, which was shown to be associated with cell cycle arrest in the G1 phase. Western blot and quantitative polymerase chain reaction analyses demonstrated that the expression levels of the anti-apoptotic gene $\mathrm{Bcl}-2$ and the $\mathrm{Bcl}-2 / \mathrm{Bax}$ ratio in the oridonin and the oridonin plus gemcitabine groups were significantly downregulated as compared with the gemcitabine treatment and control groups. The expression levels of pro-apoptotic genes Bax, cytochrome c (cyt c), and caspase-3 and -9 in the oridonin and the combination groups were significantly upregulated as compared with the other two groups. The results suggested that oridonin improved the anti-tumor effects of gemcitabine
\end{abstract}

Correspondence to: Professor Hai Huang, Department of General Surgery, Guangxing Affiliated Hospital of Zhejiang Chinese Medical University, No. 453 Ti-Yu-Chang Road, Hangzhou, Zhejiang 310007, P.R. China

E-mail: 13738050750@163.com

${ }^{*}$ Contributed equally

Key words: oridonin, gemcitabine, apoptosis, Bcl-2, Bax, cytochrome $\mathrm{C}$, caspases through the enhancement of gemcitabine-induced apoptosis. This mechanism may be through the downregulation of Bcl-2 expression and the upregulation of Bax expression, resulting in the reduction of the $\mathrm{Bcl}-2 / \mathrm{Bax}$ ratio. These effects may promote the release of cyt $\mathrm{c}$ from the mitochondria into the cytoplasm thus triggering the mitochondrial apoptosis signaling pathway. Furthermore, caspase- 3 and -9 were shown to be activated as a result of the induction of apoptosis.

\section{Introduction}

Pancreatic cancer is one of the major causes of cancer-associated deaths worldwide. Pancreatic cancer is the fourth leading cause of cancer deaths in the United States, with a median survival time of 4-6 months, and an overall 5 year survival rate of $\sim 6 \%(1,2)$. Pancreatic cancer is associated with a fast progression and a poor prognosis, and has an increasing incidence. Early diagnosis of pancreatic carcinoma is currently difficult, and the majority of patients are not diagnosed until an advanced stage $(3,4)$. Furthermore, pancreatic cancer is highly resistant to apoptosis-inducing therapy, such as chemotherapy (5).

Gemcitabine is currently the standard chemotherapeutic first line drug used to treat patients with pancreatic cancer (6); however, clinical trials have confirmed that gemcitabine chemotherapy alone is unlikely to be successful $(7,8)$. Previous evidence from laboratory studies have suggested that combinations of chemopreventive agents may be more effective for the treatment of cancer as compared with any single constituent. Previous studies have identified numerous types of drugs that can strengthen the therapeutic effects of gemcitabine in pancreatic cancer treatment (9-13). However, currently there are no convincing results on clinically relevant improvements in quality of life and survival. Therefore, it is imperative to find a drug which can potentiate the effects of gemcitabine, which has a low toxic effect and is relatively inexpensive.

Oridonin, an ent-kaurane diterpenoid isolated from Rabdosia rubescens, is a traditional Chinese herbal remedy, which has multiple biological activities, including anti-inflammatory, anti-bacterial and anti-tumor effects (14). Previous studies have demonstrated that oridonin can inhibit cell growth 
and induce apoptosis in several types of tumor cells. Apoptosis can be triggered in numerous ways, and it is well known that various proteins, including the cysteine-dependent aspartate-specific proteases (caspase), B cell lymphoma-2 (Bcl-2), and mitogen-activated protein kinase (MAPK) families and the phosphoinositide 3-kinase signal transduction pathway have important roles in the regulation of the apoptotic process (15-17). A previous study demonstrated that oridonin may potentiate the therapeutic effects of gemcitabine against pancreatic carcinoma cells by upregulating p38 and its downstream target $\mathrm{p} 53$, leading to the inhibition of proliferation, the induction of apoptosis and the suppression of invasion in vitro and in vivo (18). However, it is currently unknown whether the anti-tumor effects of the combination treatment of gemcitabine and oridonin in pancreatic cancer, is due to involvement of the mitochondrial signaling pathway.

The aim of the present study was to investigate the effects of oridonin on the growth of PANC-1 human pancreatic cancer cells in culture, and examine the effects of oridonin in combination with gemcitabine in vitro. The role of apoptosis-related proteins in the induction of apoptosis in the PANC-1 cells was also determined. It was demonstrated that oridonin could significantly enhance the anti-tumor effects of gemcitabine on pancreatic cancer in vitro, via the mitochondrial signaling pathway.

\section{Materials and methods}

Chemicals and reagents. Oridonin was obtained from the Beijing Institute of Biological Products (Beijing, China). The purity of the oridonin (99.4\%) was measured using high performance liquid chromatography. Oridonin was dissolved in dimethyl sulfoxide (DMSO) to make a stock solution at 10 mmol-1 concentration, which was stored at $-20^{\circ} \mathrm{C}$, until further use. The DMSO concentration was kept below $0.1 \%$ in all of the cell cultures, and did not exert any detectable effects on cell growth or death. Gemcitabine ${ }^{\circledR}$ (Gemzar) was obtained from Eli Lilly (Indianapolis, IN, USA) and was stored at $4^{\circ} \mathrm{C}$ and dissolved in sterile phosphate-buffered saline (PBS) at 0.2 mmol-1 concentration. The following reagents were obtained from numerous companies, as detailed: Fetal bovine serum (FBS), trypsin containing EDTA, RPMI-1640, Cell Counting kit-8 (CCK-8) (Gibco-BRL, Carlsbad, CA, USA), Annexin V-fluorescein isothiocyanate-propidium iodide (FITC-PI) Apoptosis Detection kit (Biological Development Co, Ltd. Nanjing KGI, China), Bicinchoninic Acid (BCA) Protein Assay kit (Pik-day Institute of Biotechnology, Haimen, China), RNA Extraction kit (Life Technologies, Carlsbad, CA, USA), cDNA First Strand Synthesis kit (Fermentas, Thermo Scientific, Waltham, MA, USA), $2 \times$ Taq Polymerase Chain Reaction (PCR) MasterMix (TIANGEN, Beijing, China). Ribonuclease A (RNase A), PI, DMSO (Sigma-Aldrich, St. Louis, MO, USA). Antibodies against Bcl-2, Bax, cytochrome c, caspase-9, caspase-3, $\beta$-actin and horseradish peroxidase (HRP)-conjugated secondary antibodies (goat-anti-rabbit and goat-anti-mouse) were purchased from Sigma-Aldrich.

Cell culture. The PANC-1 cells were obtained from the American Type Culture Collection (Manassas, VA, USA).
The cells were cultured in RPMI-1640 supplemented with $10 \%$ FBS, 100 units $^{-} \mathrm{mL}$ penicillin, and $100 \mu \mathrm{g}^{-} \mathrm{mL}$ streptomycin (Gibco-BRL, Grand Island, NY, USA). The cells were maintained at $37^{\circ} \mathrm{C}$ in a humidified atmosphere with $5 \% \mathrm{CO}_{2}$. The medium was changed every 2-3 days, and the cells were subcultured when confluency reached $70-80 \%$ by $0.25 \%$ trypsin digestion at $37^{\circ} \mathrm{C}$.

Cell proliferation assay. Cell proliferation was determined using the CCK-8 kit. Briefly, PANC-1 cells in the logarithmic phase of growth were plated in 96-well culture plates $\left(5 \times 10^{3}\right.$ cells per well). Following $24 \mathrm{~h}$ incubation, the cells were treated with vehicle alone $(0.1 \% \mathrm{DMSO})$ or various concentrations of oridonin $(20,40,60,80,100 \mu \mathrm{M})$, followed by a 24 , 48 , or 72 h cell culture. Each group had 6 wells of cells. CCK-8 reagent $(100 \mu \mathrm{l})$ was added to each well $1 \mathrm{~h}$ prior to the end of the incubation. The absorbance of each well was read at $450 \mathrm{~nm}$ using an ELISA reader (ELx808; Bio-Tek, Winooski, VT, USA). The experiments were repeated three times. To determine the inhibition rate of the drugs on the cells, the following calculation was performed:

Inhibition Rate $(\%)=1$-(dosing absorbance-blank absorbance)-(control absorbance-blank absorbance) $\times 100 \%$.

Cell viability assay. PANC-1 cells were seeded at a density of $5 \times 10^{3} /$ well in 96-well plates overnight and treated with $20 \mu \mathrm{M}$ of gemcitabine and/or $40 \mu \mathrm{M}$ of oridonin for $48 \mathrm{~h}$. The experiment was set up in triplicate. Untreated cells in medium alone, were used as a control. The cell viability was determined using the CCK-8 kit, according to the manufacturer's instructions. The viability of the control cells was designated as $100 \%$, and the viability of the experimental groups was calculated relative to the control.

Cell apoptosis assay. Apoptosis induction was assessed using the Annexin V-FITC kit, according to the manufacturer's instructions. PANC-1 cells were exposed to control (DMSO only), or a specific concentration of oridonin $(40 \mu \mathrm{M})$ and gemcitabine $(20 \mu \mathrm{M})$ alone or in combination for $48 \mathrm{~h}$ in 6 -well plates. The floating and adherent cells were collected by centrifugation at $1,000 \mathrm{x}$ g for $5 \mathrm{~min}$.The pooled cells were washed three times with cold PBS. Subsequently, $\sim 1 \times 10^{5}$ cells were resuspended in $100 \mu \mathrm{l}$ of binding buffer, and mixed with $5 \mu \mathrm{l}$ of Annexin V-FITC and $10 \mu \mathrm{l}$ of PI. Following $15 \mathrm{~min}$ of incubation in the dark at room temperature, the fluorescence intensities of $>10,000$ viable cells from each sample were analyzed using a COULTER ${ }^{\circledR}$ EPICS $^{\circledR}$ XL $^{\mathrm{TM}}$ Flow Cytometer (Beckman Coulter, Miami, FL, USA) with excitation and emission settings of 488 and $525 \mathrm{~nm}$, respectively. The experiments were repeated three times. The data were analyzed using CellQuest ${ }^{\mathrm{TM}}$ (BD Biosciences, Franklin Lakes, NJ, USA) software.

Cell cycle analysis. The effects of oridonin on the distribution of the cell cycle were determined by flow cytometric analysis of the DNA content from the nuclei of the cells, following PI staining. Asynchronized (70-80\%) confluent cells were treated with 20,40 or $80 \mu \mathrm{M}$ oridonin for $48 \mathrm{~h}$. Control cells were treated with $0.1 \%$ DMSO only. Following incubation of the cells at $37^{\circ} \mathrm{C}$ for the specified time, suspension and adherent 
Table I. Primer pairs used in quantitative polymerase chain reaction.

\begin{tabular}{llc}
\hline Gene & \multicolumn{1}{c}{ Primer pairs (5'-3') } & Product size (bp) \\
\hline Bcl-2 & S: AGCCGGGAGAACAGGGTATG & A: ATCCAGGTGTGCATGCCG \\
Bax & S: ATGGCTGGGGAGACACCTGA & 549 \\
Ayt-C & A: TGGGCGTCCCGAAGTAGGAA & 394 \\
Caspase-9 & A: GCGTGTCCTTGGACTTAGAG & 242 \\
Caspase-3 & S: GGTTCTGGAGGATTTGGTGA & 325 \\
GAPDH & A: GACAGCCGTGAGAGAGAATGA & 309 \\
& S: AGCAAACCTCAGGGAAACATT & \\
& A: GTCTCAATGCCACAGTCCAGT & 216 \\
\hline
\end{tabular}

Bp, base pairs; S, sense; A, antisense.

cells were collected using $0.05 \%$ trypsin, washed twice with cold PBS and fixed with ice-cold $70 \%$ ethanol overnight at $4^{\circ} \mathrm{C}$. The fixed cells were centrifuged at $300 \mathrm{x} \mathrm{g}$ for $10 \mathrm{~min}$, followed by washing with PBS. Subsequently, the cells were treated with $80 \mathrm{mg}^{-} \mathrm{ml}$ RNase A for $30 \mathrm{~min}$ at $37^{\circ} \mathrm{C}$. The cells were chilled in ice for $10 \mathrm{~min}$ and stained with PI $(50 \mathrm{mg}-\mathrm{ml}$ final concentration) for $1 \mathrm{~h}$ in the dark. The stained cells were analyzed using a COULTER EPICS XL Flow Cytometer. A total of $\sim 20,000$ cells were evaluated for each experiment. In all evaluations, cell debris and cell clumps were excluded from the analysis. The cell cycle data were analyzed using ModFit (Verity Software House, Topsham, ME, USA) software.

Western blot analysis. The PANC-1 cells were incubated with an allocated concentration of oridonin $(40 \mu \mathrm{M})$ and gemcitabine $(20 \mu \mathrm{M})$, either alone or in combination for $48 \mathrm{~h}$. The cells cultured in medium alone were used as controls. Cytoplasmic protein was gathered using the mitochondrial-cytoplasmic protein isolation kit (Sigma, Carlsbad, CA, USA) and cytochrome c levels were detected according to the manufacturer's instructions. Subsequently, cells were harvested and washed twice with ice-cold PBS, the cell pellets were resuspended in lysis buffer consisting of $50 \mathrm{mM}$ Hepes (pH 7.4), 1\% Triton-X 100, 2 mM sodium orthovanada, $100 \mathrm{mM}$ sodium fluoride, $1 \mathrm{mM}$ edetic acid, $1 \mathrm{mM}$ phenylmethanesulfonyl fluoride, $10 \mathrm{mg}^{-1}$ aprotinin, and $10 \mathrm{mg}^{-1}$ leupeptin (Sigma-Aldrich) and lysed at $4^{\circ} \mathrm{C}$ for $60 \mathrm{~min}$. After $13,000 \times \mathrm{g}$ centrifugation for $15 \mathrm{~min}$, the protein content of the supernatant was determined by BCA assay according to the manufacturer's instructions. The protein lysates (20 $\mu \mathrm{g}$-lane) were separated by $12 \%$ SDS-PAGE, followed by transfer to a nitrocellulose membrane (Pierce Biotechnology, Inc., Rockford, IL, USA). Each membrane was blocked with $5 \%$ skim milk and incubated with the indicated primary antibodies against Bcl-2, Bcl-2 associated $\mathrm{X}$ protein (Bax), cytochrome $\mathrm{c}$ (cytosol), caspase- 9 and caspase- 3 , and $\beta$-actin overnight at $4{ }^{\circ} \mathrm{C}$. The membrane was then incubated with goat anti-rabbit and goat anti-mouse immunoglobulin $\mathrm{G}$ conjugated with HRP for $1 \mathrm{~h}$ at room temperature. The immunocomplexes were observed using an enhanced chemiluminescence reagent followed by visualization on X-ray films. The quantitative data are expressed as a percentage of the means \pm standard deviation of the relative levels of the objective protein and control $\beta$-actin of each group of cells, from three independent experiments.

Quantitative PCR ( $q$ PCR). The PANC-1 cells were treated with an assigned concentration of oridonin $(40 \mu \mathrm{M})$ and gemcitabine $(20 \mu \mathrm{M})$, either alone or in combination, for $48 \mathrm{~h}$. The control cells were treated with $0.1 \%$ DMSO only. Total cellular RNA was isolated from the cells using TRIzol ${ }^{\circledR}$ reagent (Invitrogen Life Technologies, Carlsbad, CA, USA), and the RNA content was measured using a UV spectrophotometer (Bio-Rad, Hercules, CA, USA) at $260 \mathrm{~nm}$. cDNA was synthesized from $1 \mu \mathrm{g}$ of total RNA and oligo dT primers, according to the manufacturer's instructions. The following PCR amplification conditions were used: Bcl-2, $94^{\circ} \mathrm{C} 20 \mathrm{~s}$, $58^{\circ} \mathrm{C} 20 \mathrm{~s}, 72^{\circ} \mathrm{C} 20 \mathrm{~s}, 35$ cycles; Bax, $94^{\circ} \mathrm{C} 30 \mathrm{~s}, 57^{\circ} \mathrm{C} 30 \mathrm{~s}$, $72^{\circ} \mathrm{C} 20 \mathrm{~s}, 30$ cycles; cytochrome c, $94^{\circ} \mathrm{C} 30 \mathrm{~s}, 60^{\circ} \mathrm{C} 30 \mathrm{~s}$, $72^{\circ} \mathrm{C} 30 \mathrm{~s}, 35$ cycles; caspase $-9,94^{\circ} \mathrm{C} 30 \mathrm{~s}, 56^{\circ} \mathrm{C} 30 \mathrm{~s}, 72^{\circ} \mathrm{C}$ $30 \mathrm{~s}, 35$ cycles; caspase- $3,94^{\circ} \mathrm{C} 30 \mathrm{~s}, 57^{\circ} \mathrm{C} 30 \mathrm{~s}, 72^{\circ} \mathrm{C} 30 \mathrm{~s}, 35$ cycles; GAPDH, $94^{\circ} \mathrm{C} 30 \mathrm{~s}, 54^{\circ} \mathrm{C} 30 \mathrm{~s}, 72^{\circ} \mathrm{C} 20 \mathrm{~s}, 25$ cycles. GAPDH was used as an internal control. The primer pairs used for the amplification are listed in Table 1. A total of $5 \mu \mathrm{l}$ of the PCR product was separated on a $1 \%$ agarose gel the results were visualized.

Statistical analysis of the data. All results were repeated in at least three separate experiments. The data are expressed as the means \pm standard deviation. Statistical comparisons were made using a one-way analysis of variance, which revealed significant differences between groups, and a Student's t-test which revealed significant differences between two sample means. Statistical analyses were carried out using SPSS version 17.0 software (SPSS Inc., Chicago, IL, USA). A $\mathrm{P}<0.05$ was considered to indicate a statistically significant difference. 


\section{Results}

Oridonin suppresses proliferation and potentiates growth inhibition induced by gemcitabine, in pancreatic cancer cells. The chemical structure of oridonin can be seen in Fig. 1A. The effects of oridonin on the proliferation of the PANC-1 cells were determined using the CCK-8 assay (Fig. 1B). Oridonin was shown to inhibit the growth of the cells in a dose- and time-dependent manner. The half maximal inhibitory concentration of oridonin was determined as $38.86 \mu \mathrm{M}$ for $48 \mathrm{~h}$ treatments. The cell viability was evaluated by CCK- 8 assay, and the results showed that the combination treatment of oridonin plus gemcitabine resulted in an increased loss of cell viability as compared with either oridonin or gemcitabine treatment alone in PANC-1 cells $(\mathrm{P}<0.05)$ (Fig. 2).

Oridonin potentiates the apoptotic effects of gemcitabine in pancreatic cancer cells in vitro. To determine whether oridonin enhances gemcitabine-induced apoptosis, the presence of apoptotic cells was observed by flow cytometric analysis of Annexin V-FITC-PI stained PANC-1 cells. The cells were treated with oridonin $(40 \mu \mathrm{M})$ and gemcitabine $(20 \mu \mathrm{M})$, either alone or in combination for $48 \mathrm{~h}$. It was observed that oridonin and gemcitabine alone were minimally effective in increasing the apoptotic rate $(\mathrm{P}<0.05)$, whereas the combination of gemcitabine and oridonin significantly increased the rate of apoptosis, as compared with the single-agent treatments $(\mathrm{P}<0.01)$ (Fig. $3 \mathrm{~A}$ and $\mathrm{B})$. These data are consistent with results from the cell growth inhibition studies using CCK-8, suggesting that the reduction in the number of viable cells by oridonin and/or gemcitabine, is in part due to the induction of cell apoptosis.

Oridonin synergizes the apoptotic effects of gemcitabine via G0/G1 phase arrest in PANC-1 cells. To further investigate whether the effects of oridonin on gemcitabine-induced apoptosis were correlated with cell cycle arrest, the DNA contents of PANC-1 cells, treated with 20,40 and $80 \mu \mathrm{M}$ oridonin or vehicle ( $0.1 \%$ DMSO treated) for $48 \mathrm{~h}$, were analyzed using a flow cytometer. Following oridonin treatment, cell cycle analysis revealed that oridonin increased the number of cells in the G0/G1 cell cycle phase, with a concomitant decrease observed in the number of treated cells in the $\mathrm{S}$ and $\mathrm{G} 2$ phases, in a dose-dependent manner as compared with the control $(\mathrm{P}<0.05)$ (Fig. 4).

Effects of oridonin and gemcitabine on the expression of apoptosis-related proteins in PANC-1 cells. Two principle apoptosis signaling pathways have been previously identified: The death receptor pathway (extrinsic pathway) and the mitochondrial pathway (intrinsic pathway) (19). The activation of caspases is affected by the Bcl-2 family proteins, which have an important role in the intrinsic pathway. A decreased Bcl-2/Bax ratio, activation of caspases and the release of cytochrome $\mathrm{c}$ are all recognized as important events which mediate the mitochondrial apoptotic pathway. Therefore, in order to clarify how oridonin increases the rate of gemcitabine-induced apoptosis in PANC-1 cells, the changing levels of these proteins, in response to oridonin and gemcitabine treatments, were determined using western blot analysis. The PANC-1
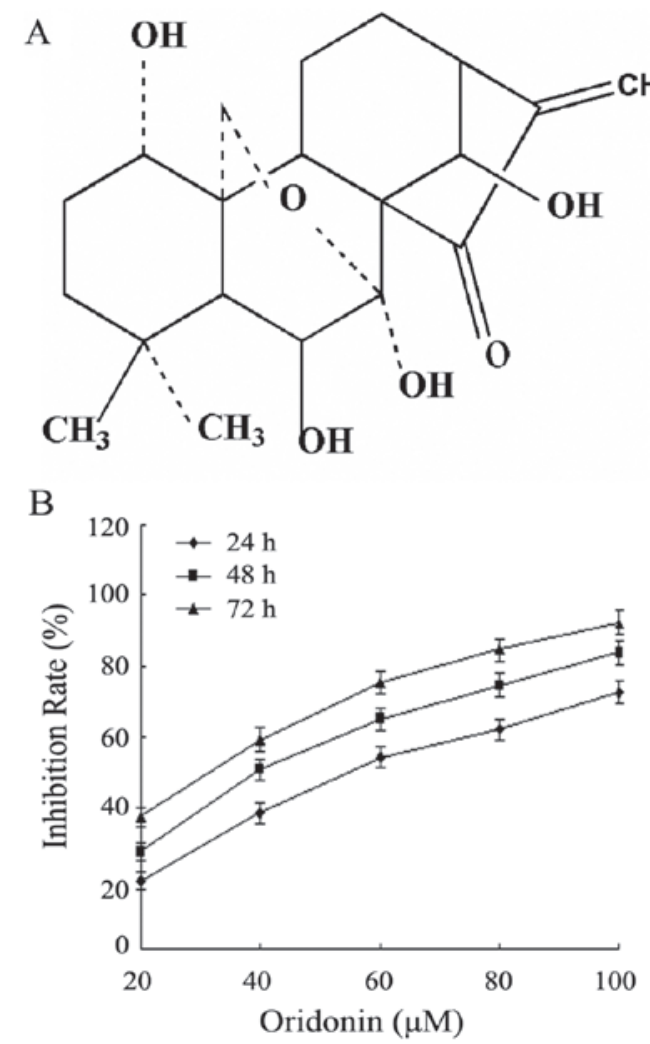

Figure 1. Effects of oridonin on inhibiting the growth of PANC-1 pancreatic cancer cells in vitro. (A) The chemical structure of oridonin. (B) Panc-1 cells were treated with vehicle, or $20,40,60,80$ or $100 \mu \mathrm{M}$ of oridonin for 24 48 , or $72 \mathrm{~h}$. The cell proliferation inhibition rates were determined by Cell Counting kit- 8 assay, and the data are presented as the means \pm standard deviation from three independent experiments.

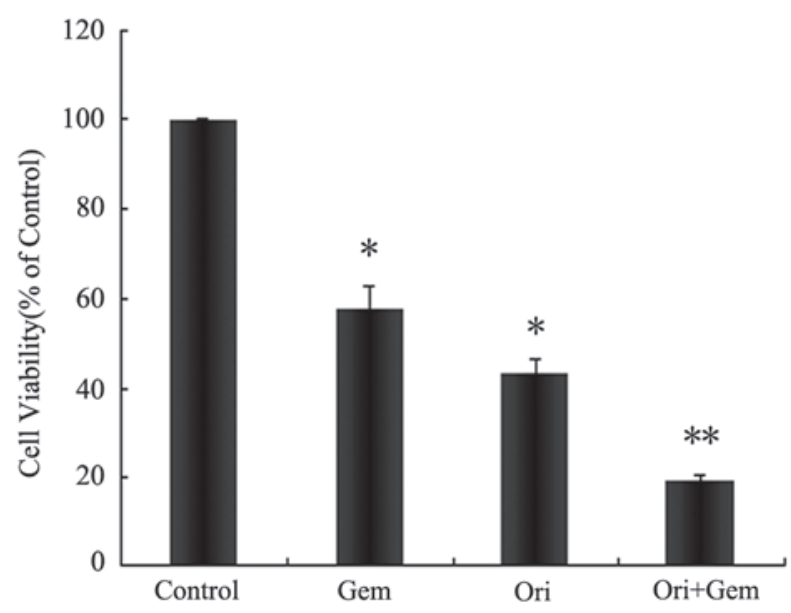

Figure 2. PANC-1 pancreatic cancer ells were treated with oridonin $(40 \mu \mathrm{M})$ and gemcitabine $(20 \mu \mathrm{M})$, either alone or in combination for $48 \mathrm{~h}$ and cell viability was analyzed by the Cell Counting kit- 8 assay. Data are presented as the means \pm standard deviations from three independent experiments. ${ }^{*} \mathrm{P}<0.05$ vs. the control; ${ }^{* *} \mathrm{P}<0.05$ vs. the control, oridonin or gemcitabine treatment. Gem, gemcitabine; Ori, oridonin.

cells were treated with oridonin and gemcitabine, alone or in combination, for $48 \mathrm{~h}$. As shown in Fig. 5, both oridonin alone, or in combination with gemcitabine, downregulated the protein expression of $\mathrm{Bcl}-2$, and increased the protein expressions of Bax, cytochrome c (cytosol), caspase- 9 and -3 , 

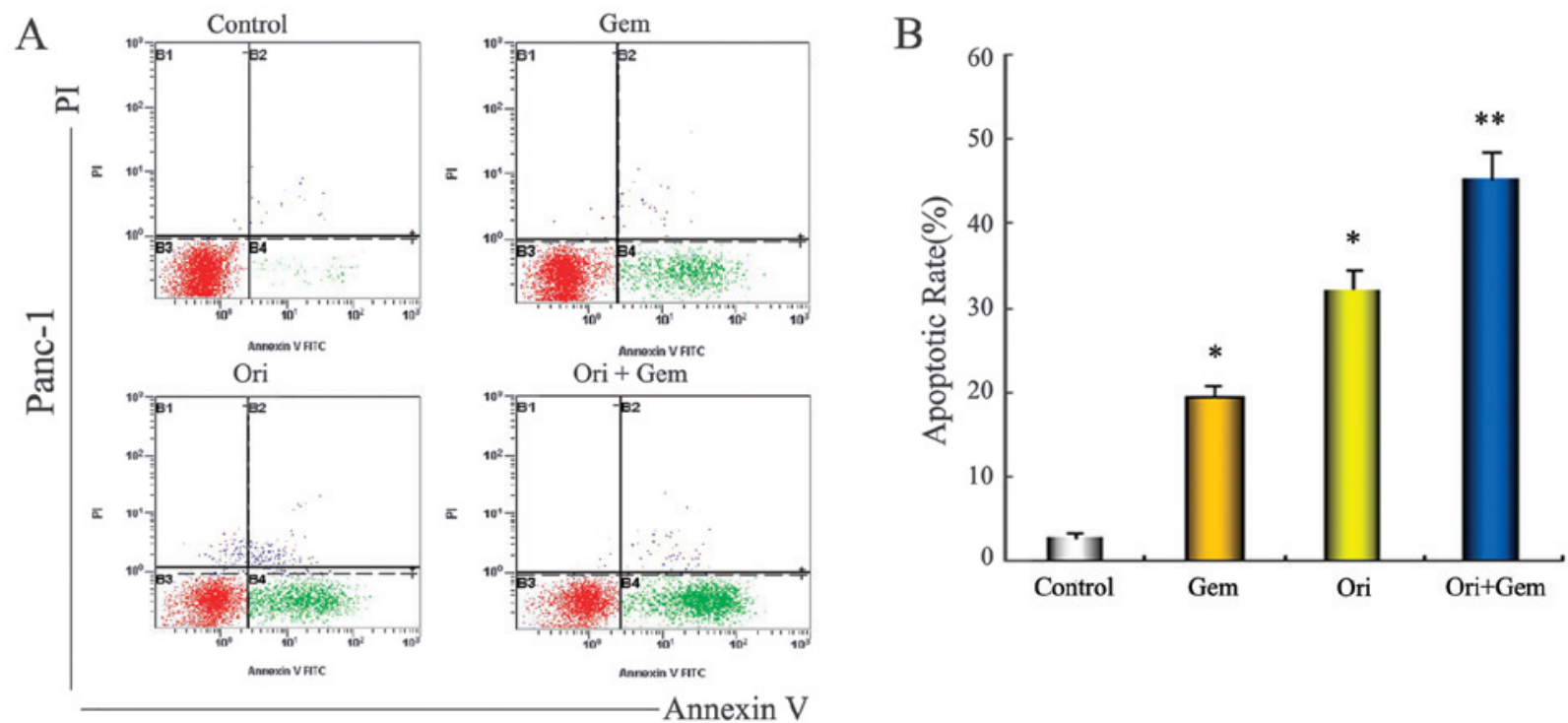

Figure 3. Effects of oridonin on the levels of apoptosis in PANC-1 pancreatic cancer cells in vitro. (A) PANC-1 cells treated with oridonin (40 $\mu \mathrm{M})$ and gemcitabine $(20 \mu \mathrm{M})$ alone or in combination for $48 \mathrm{~h}$ were measured by dual dye staining using annexin V-fluorescein isothiocyanate/propidium iodide. The results are representative of three independent experiments. (B) The percentage of apoptotic cells are presented as the means \pm standard deviation of three independent experiments. ${ }^{*} \mathrm{P}<0.05,{ }^{* *} \mathrm{P}<0.01$ vs. the control or the cells treated with gemcitabine. Gem, gemcitabine; Ori, oridonin; FITC, fluorescein isothiocyanate;PI, propidium iodide.

A
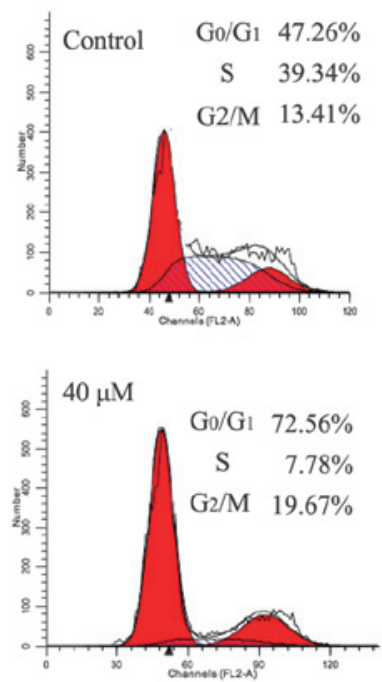
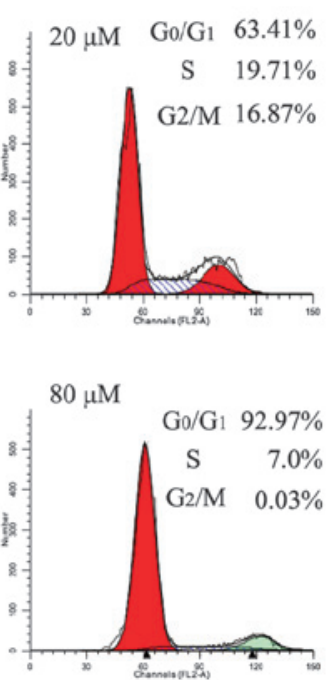

B

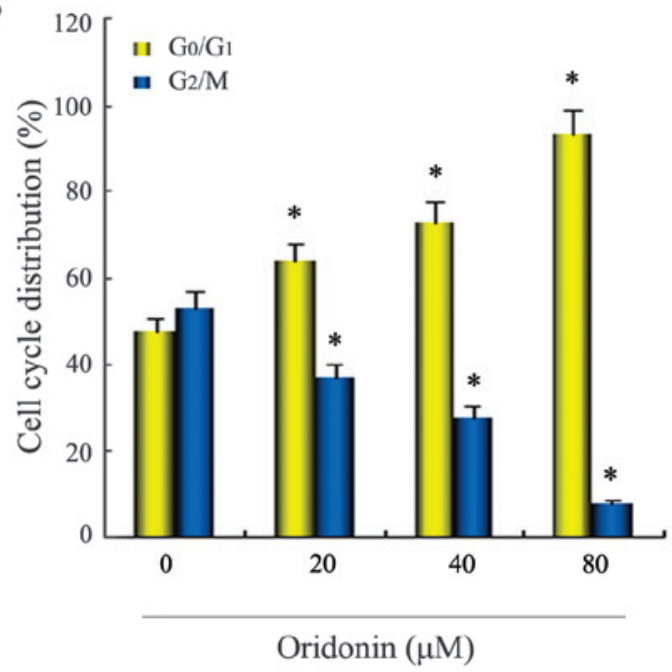

Figure 4. Effects of oridonin on the distribution of PANC-1 pancreatic cancer cells across phases of the cell cycle. (A) PANC-1 cells were treated with 20,40 or $80 \mu \mathrm{M}$ oridonin for $48 \mathrm{~h}$ and the control cells were treated with $0.1 \%$ dimethyl sulfoxide. The cell cycle distribution was assessed by flow cytometry. The results are representative of three independent experiments. (B) The cell cycle distribution was determined using ModFit software version 3.1. The proportions of cells in the G1, and S plus G2 phase are presented as the means \pm standard deviation of three independent experiments. ${ }^{*} \mathrm{P}<0.05$ vs. the control.

as compared with the expression levels of the control cells $(\mathrm{P}<0.05)$. Furthermore, combination therapy promoted the reduction of the $\mathrm{Bcl}-2 / \mathrm{Bax}$ ratio $(\mathrm{P}<0.05)$ as compared with monotherapy with either agent. These results suggested that the improvement of gemcitabine-induced apoptosis by the addition of oridonin was mainly achieved through activation of the mitochondrial apoptotic pathway.

Effects of oridonin and gemcitabine on the expression of apoptosis-related genes in vitro. Similar results to the western blot analysis were obtained from the qPCR. As shown in Fig. 6, as compared with the control, oridonin treatment alone or in combination with gemcitabine both downregulated the mRNA expression levels of $\mathrm{Bcl}-2(\mathrm{P}<0.05)$, and upregulated the expression levels of Bax, cytochrome c, caspase- 9 and -3 (all $\mathrm{P}<0.05$ ). The changes to the expression levels were more evident in the combination of oridonin and gemcitabine group, as compared with the other groups. $(\mathrm{P}<0.05)$. Similarly, the results also showed a marked reduction in the Bcl-2-Bax ratio when the PANC-1 cells were treated with a combination of oridonin and gemcitabine, as compared with the other groups. These results were in line with the protein expression changes and provided further proof for the mechanism of action of oridonin. 

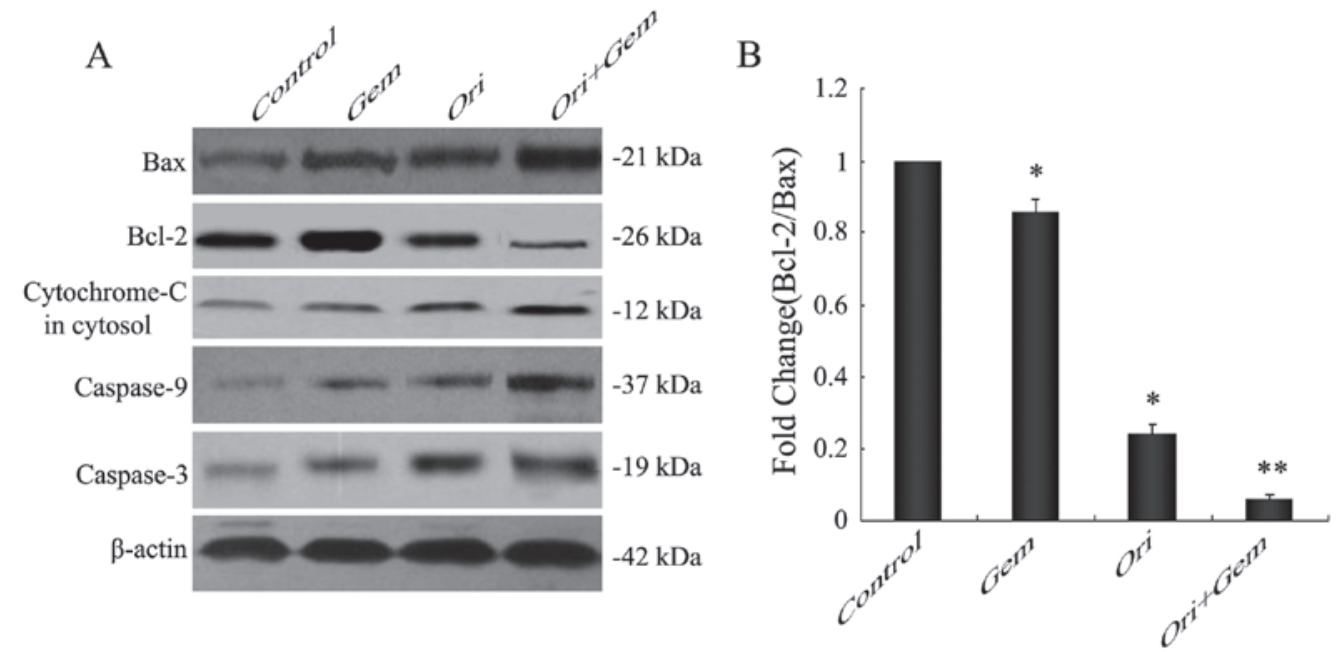

$\mathrm{C}$

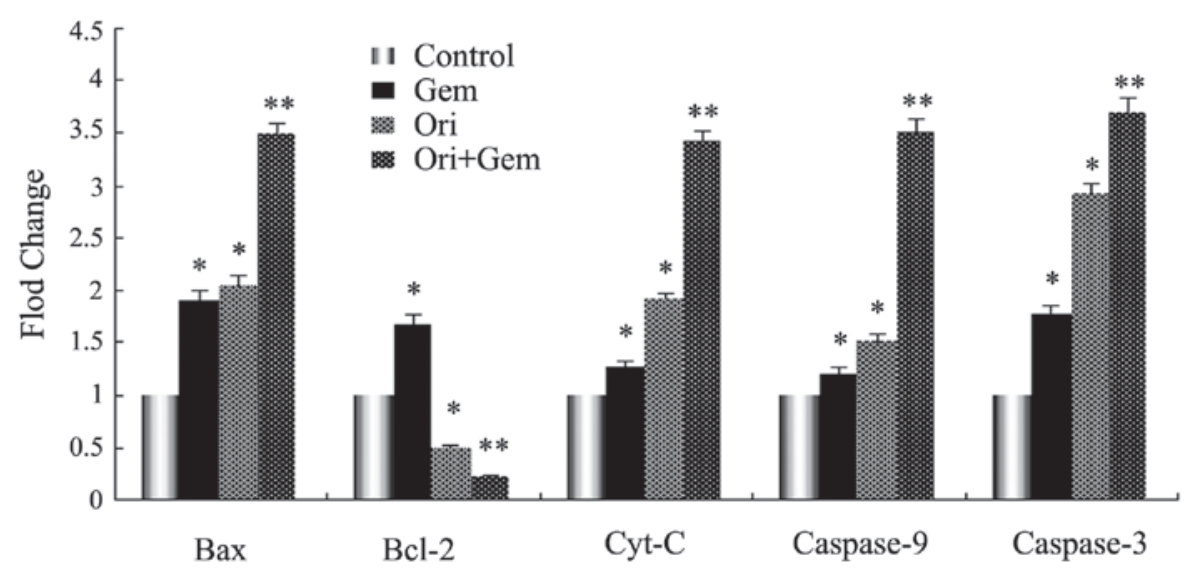

Figure 5. Oridonin potentiates the effects of gemcitabine in PANC-1 pancreatic cancer cells by upregulating Bax and downregulating Bcl-2 expression, reducing the Bcl-2/Bax ratio, promoting cytochrome c release and activating caspase- 9 and -3 in vitro. The cells were treated with oridonin (40 $\mu \mathrm{M})$ and gemcitabine $(20 \mu \mathrm{M})$ alone or in combination for $48 \mathrm{~h}$. The cell lysates were separated by $12 \%$ SDS-PAGE, and the protein expressions were detected by western blot analysis. The data are presented as the means \pm standard deviation, $n=3$. (A) Oridonin alone and combined with gemcitabine was observed to downregulate the expression of Bcl-2, and upregulate the expression of Bax, cytochrome c (cytosol), and caspases-9 and -3 . (B and C) The control group data were normalized to 1 for the quantification. ${ }^{*} \mathrm{P}<0.05$ vs. control; ${ }^{* *} \mathrm{P}<0.05$ vs. control or gemcitabine alone group. Gem, gemcitabine; Ori, oridonin; $\mathrm{kDa}$, kilodalton.

\section{Discussion}

In recent years, research using natural substances to enhance the chemotherapeutic effects of gemcitabine has become more widespread (20). Previous studies have demonstrated that natural compounds can enhance the therapeutic effects of gemcitabine on pancreatic cancer (21-25). Oridonin has been shown to increase the sensitivity of apoptosis-inducing drugs, including imatinib and arsenic trioxide $(26,27)$. A previous study showed that oridonin significantly enhanced the anti-tumor and pro-apoptotic effects of gemcitabine on the BXPC-3 pancreatic cancer cell line (28). In the present study, the data presented indicated that varying concentrations of oridonin effectively inhibited the proliferation of the PANC-1 cell line at different time points, and found that oridonin enhanced the apoptotic effects of gemcitabine in PANC-1 cells in vitro. Gemcitabine alone had a minimal effect on apoptosis in the PANC-1 cell line, however, the combination of oridonin and gemcitabine was observed to be highly effective in inducing apoptosis. Oridonin was shown to augment the apoptotic effects of gemcitabine, through G1 phase cell cycle arrest. The present study was the first, to the best of our knowledge, to determine that oridonin is capable of sensitizing PANC-1 cells to gemcitabine in vitro through the alteration of expression levels of apoptotic factors. Oridonin was shown to induce a reduction in the $\mathrm{Bcl}-2 / \mathrm{Bax}$ ratio, increasing the release of cytochrome $\mathrm{c}$ from the mitochondria into the cytosol, and resulting in the concomitant activation of caspase- 3 and 9 in the mitochondrial apoptosis signaling pathway.

The mitochondrial apoptotic pathway is regulated by the Bcl-2 family of proteins, including the anti-apoptotic proteins $\mathrm{Bcl}-2$ and $\mathrm{Bcl}-\mathrm{xL}$, the pro-apoptotic proteins Bax and $\mathrm{Bcl}-2$ homologous antagonist killer (Bak) and the apoptosis initiator proteins $\mathrm{Bcl}-2$ assocoated death promote (Bad) and Bcl-2 interacting killer (Bik). The accumulation of pro-apoptotic proteins on the mitochondrial outer membrane results in increased mitochondrial membrane permeability, causing the release of cytochrome $\mathrm{c}$ into the cytoplasm. Cytochrome $\mathrm{c}$ promotes the activation of caspase- 9 , which in turn promotes the activation of caspase-3, leading to apoptosis of the tumor cell (29-31). An important function of the mitochondria in apoptotic signaling is the translocation of cytochrome $\mathrm{c}$ from the mitochondrial 

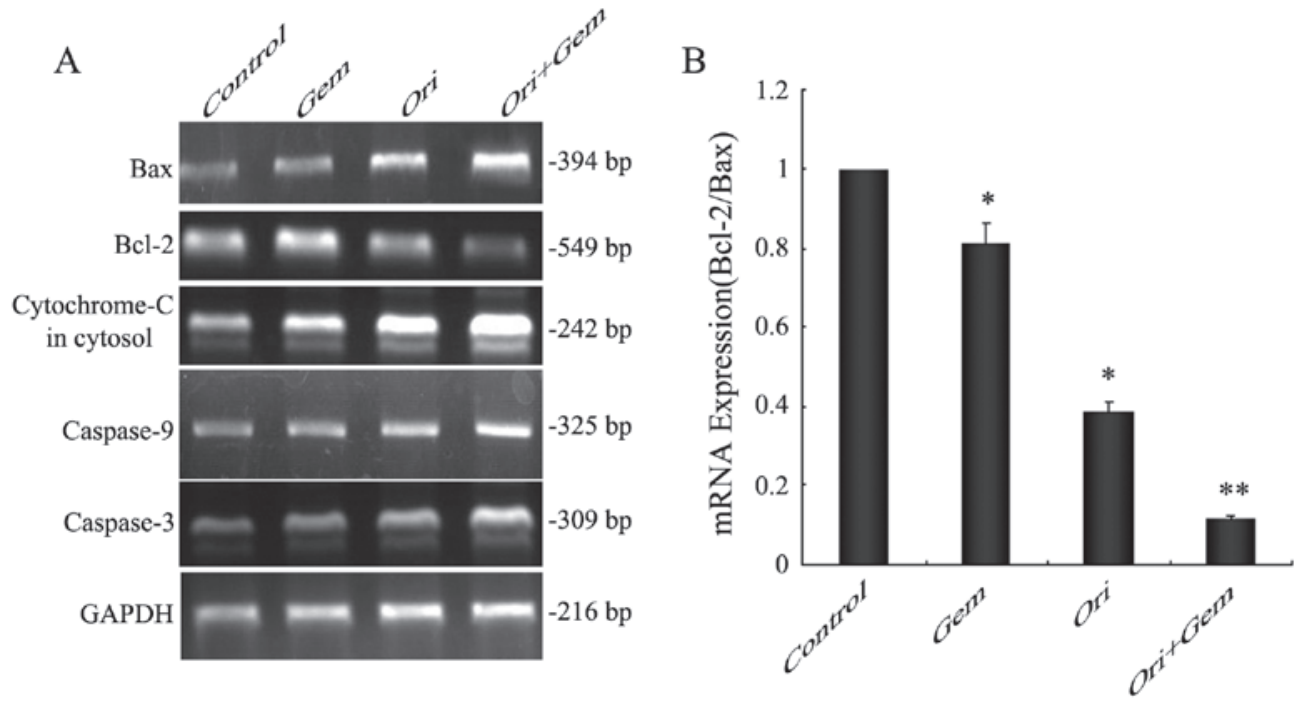

C

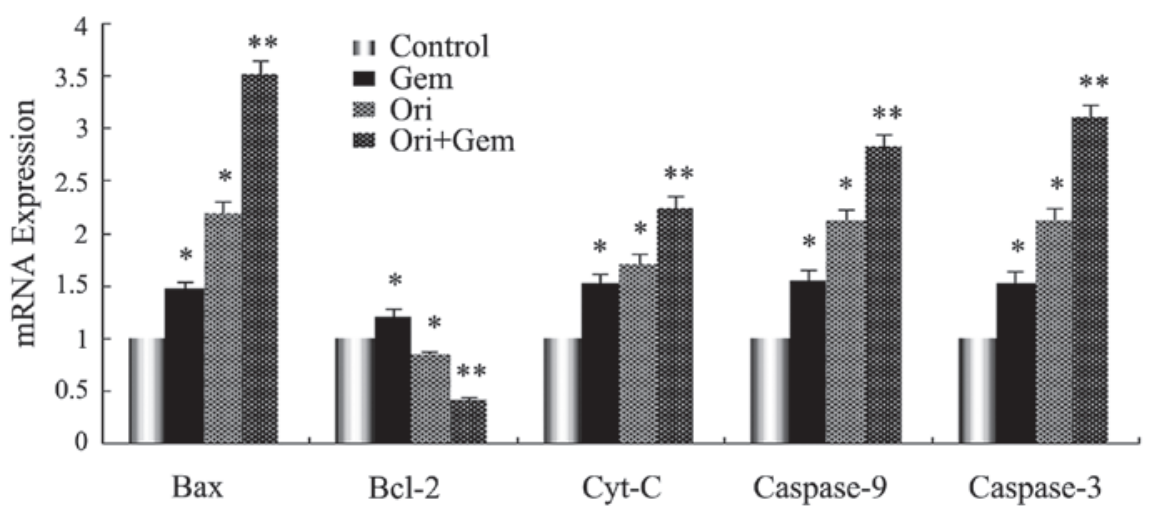

Figure 6. Effects of oridonin and gemcitabine on the mRNA expression levels of Bcl-2, Bax, cytochrome C (cytosol) and caspase-9 and -3 in PANC-1 pancreatic cancer cells in vitro, as measured by quantitative polymerase chain reaction. (A) Oridonin alone and combined with gemcitabine was observed to downregulate the expression of Bcl-2, and upregulate the expressions of Bax, cytochrome c (cytosol) and caspases-9 and -3. (B and C) TThe control group data were normalized to 1 for the quantification.. ${ }^{*} \mathrm{P}<0.05$ vs. control; ${ }^{* *} \mathrm{P}<0.05$ vs. control or cells treated with gemcitabine alone. Gem, gemcitabine; Ori, oridonin; bp, base pairs.

intermembrane compartment, into the cytosol. The release of cytochrome c, and cytochrome c-mediated apoptosis, is controlled prominently by members of the Bcl-2 family, of which Bax and Bcl-2 have previously been identified as major regulators. In response to a variety of stimuli, including anti-cancer drugs, Bax translocates to the mitochondria and inserts into the outer mitochondrial membrane, allowing the release of cytochrome $\mathrm{c}$ into the cytoplasm. In contrast, $\mathrm{Bcl}-2$ blocks cytochrome $\mathrm{c}$ efflux by binding to the outer mitochondrial membrane and forming a heterodimer with Bax, resulting in the neutralization of its pro-apoptotic effects (32). The balance between the levels of $\mathrm{Bcl}-2$ and $\mathrm{Bax}$ is therefore critical in determining the cell fate. Furthermore, $\mathrm{Bcl}-2$ and Bax respectively suppress and promote cytochrome c release from the mitochondria, therefore the present study aimed to we further investigate the variation to the $\mathrm{Bcl}-2 / \mathrm{Bax}$ ratio. It was observed that the Bcl-2/Bax ratio was markedly reduced in PANC-1 cells following combination treatment, as revealed by western blotting and qPCR. These results demonstrate that oridonin is capable of enhancing the pro-apoptotic activity of gemcitabine in PANC-1 cells by reducing the Bcl-2/Bax ratio. The present study revealed that oridonin may be able to downregulate $\mathrm{Bcl}-2$ expression, upregulate Bax expression levels and trigger cytochrome c release, subsequently inducing cell apoptosis.

Caspases are known to have a pivotal role in the process of apoptosis. The two major caspase activation pathways, death receptor and mitochondrial pathways, have been previously described (33). In the death receptor pathway, apoptosis occurs upon the stimulation of death receptors in the cell surface which activate caspase-8. The mitochondrial pathway is dependent on the release of cytochrome $\mathrm{c}$ from the mitochondria into the cytosol, resulting in the activation of caspase-9. Caspase- 8 and caspase-9 will subsequently proteolytically activate downstream caspases, in particular caspase-3, which is essential for the morphological changes and the DNA fragmentation associated with apoptosis. There is also evidence that apoptosis can be induced through a caspase-independent mechanism (34). In the present study, western blot analysis and qPCR showed that oridonin upregulated the expression levels of caspase- 9 and caspase-3, this increase was most marked in the combination treatment group. Thus, the results demonstrate that oridonin, which enhances the pro-apoptotic effects of gemcitabine on pancreatic cancer is dependent on the activation of caspases, 
including capase- 9 and -3 . The present results are consistent with those from a previous study in which oridonin induced apoptosis in SW1990 pancreatic cancer cells via p53- and caspase-dependent induction of p38 MAPK (28).

In conclusion, the present study demonstrated that oridonin has significant potential for the treatment of pancreatic cancer through the potentiation of the anti-tumor effects of gemcitabine on PANC-1 cells by inducing apoptosis. It is proposed that oridonin may downregulate the expression levels of Bcl-2 and upregulate the expression of Bax in the mitochondrial apoptotic pathway, thereby reducing the $\mathrm{Bcl}-2 / \mathrm{Bax}$ ratio, and causing the release of cytochrome $\mathrm{c}$, from the mitochondria into the cytoplasm. This may lead to increased levels of apoptosis of tumor cells via activation of caspases-9 and -3 . Based on these results, further clinical and mechanistic studies are necessary to confirm these findings in patients with pancreatic cancer.

\section{Acknowledgements}

The authors would like to thank the Administration of Traditional Chinese Medicine of Zhejiang, China (grant no. 2013ZQ026) and The National Natural Science Foundation of China (grant no. 81202821) for financial support of this study.

\section{References}

1. Jemal A, Siegel R, Xu J and Ward E: Cancer statistics, 2010. CA Cancer J Clin 60: 277-300, 2010.

2. Oberstein PE and Olive KP: Pancreatic cancer: why is it so hard to treat? Therap Adv Gastroenterol 6: 321-337, 2013

3. Ikeda S, Maeshiro K, Ryu S, et al: Diagnosis of small pancreatic cancer by endoscopic balloon-catheter spot pancreatography: an analysis of 29 patients. Pancreas 38: e102-e113, 2009.

4. Klauss M, Stiller W, Fritz F, et al: Computed tomography perfusion analysis of pancreatic carcinoma. J Comput Assist Tomogr 36: 237-242, 2012.

5. Cao H, LE D and Yang LX: Current status in chemotherapy for advanced pancreatic adenocarcinoma. Anticancer Res 33: 1785-1791, 2013.

6. Burris HA III, Moore MJ, Andersen J, et al: Improvements in survival and clinical benefit with gemcitabine as first-line therapy for patients with advanced pancreas cancer: a randomized trial. J Clin Oncol 15: 2403-2413, 1997.

7. O'Reilly EM and Abou-Alfa GK: Cytotoxic therapy for advanced pancreatic adenocarcinoma. Semin Oncol 34: 347-353, 2007.

8. von Wichert G, Seufferlein T and Adler G: Palliative treatment of pancreatic cancer. J Dig Dis 9: 1-7, 2008.

9. Bader Y, Hartmann J, Horvath Z, et al: Synergistic effects of deuterium oxide and gemcitabine in human pancreatic cancer cell lines. Cancer Lett 259: 231-239, 2008.

10. Yu WD, Ma Y, Flynn G, et al: Calcitriol enhances gemcitabine anti-tumor activity in vitro and in vivo by promoting apoptosis in a human pancreatic carcinoma model system. Cell Cycle 9: 3022-3029, 2010.

11. Damaraju VL, Bouffard DY, Wong CK, et al: Synergistic activity of troxacitabine (Troxatyl) and gemcitabine in pancreatic cancer. BMC Cancer 7: 121, 2007.

12. Lee JK, Ryu JK, Yang KY, et al: Effects and mechanisms of the combination of suberoylanilide hydroxamic acid and bortezomib on the anticancer property of gemcitabine in pancreatic cancer Pancreas 40: 966-973, 2011.
13. Ahn DW, Seo JK, Lee SH, et al: Enhanced antitumor effect of combination therapy with gemcitabine and guggulsterone in pancreatic cancer. Pancreas 41: 1048-1057, 2012.

14. Tan W, Lu J, Huang M, et al: Anti-cancer natural products isolated from chinese medicinal herbs. Chin Med 6: 27, 2011

15. Rufini A and Melino G: Cell death pathology: the war against cancer. Biochem Biophys Res Commun 414: 445-450, 2011.

16. Kim EK and Choi EJ: Pathological roles of MAPK signaling pathways in human diseases. Biochim Biophys Acta 1802: 396-405, 2010.

17. Martelli AM, Nyåkern M, Tabellini G, Bortul R, Tazzari PL, Evangelisti C and Cocco L: Phosphoinositide 3-kinase-Akt signaling pathway and its therapeutical implications for human acute myeloid leukemia. Leukemia 20: 911-928, 2006.

18. Bu HQ, Luo J, Chen H, et al: Oridonin enhances antitumor activity of gemcitabine in pancreatic cancer through MAPK-p38 signaling pathway. Int J Oncol 41: 949-958, 2012.

19. Hengartner MO: The biochemistry of apoptosis. Nature 407: 770-776, 2000.

20. Wei WT, Chen H, Wang ZH, et al. Enhanced antitumor efficacy of gemcitabine by evodiamine on pancreatic cancer via regulating PI3K/Akt pathway. Int J Biol Sci 8: 1-14, 2012.

21. Banerjee S, Zhang Y, Ali S, et al: Molecular evidence for increased antitumor activity of gemcitabine by genistein in vitro and in vivo using an orthotopic model of pancreatic cancer. Cancer Res 65: 9064-9072, 2005.

22. Kunnumakkara AB, Guha S, Krishnan S, et al: Curcumin potentiates antitumor activity of gemcitabine in an orthotopic model of pancreatic cancer through suppression of proliferation, angiogenesis, and inhibition of nuclear factor-kappaB-regulated gene products. Cancer Res 67: 3853-3861, 2007.

23. Harikumar KB, Kunnumakkara AB, Sethi G et al: Resveratrol, a multitargeted agent, can enhance antitumor activity of gemcitabine in vitro and in orthotopic mouse model of human pancreatic cancer. Int J Cancer 127: 257-268, 2010.

24. Banerjee S, Kaseb AO, Wang Z et al: Antitumor activity of gemcitabine and oxaliplatin is augmented by thymoquinone in pancreatic cancer. Cancer Res 69: 5575-5583, 2009.

25. Wang ZH, Chen H, Guo HC, et al: Enhanced antitumor efficacy by the combination of emodin and gemcitabine against human pancreatic cancer cells via downregulation of the expression of XIAP in vitro and in vivo. Int J Oncol 39: 1123-1131, 2011.

26. Guo Y, Shan Q, Gong Y, et al: Oridonin in combination with imatinib exerts synergetic anti-leukemia effect in $\mathrm{Ph}+$ acute lymphoblastic leukemia cells in vitro by inhibiting activation of LYN-mTOR signaling pathway. Cancer Biol Ther 13: 1244-1254, 2012.

27. Chen G, Wang K, Yang BY, et al: Synergistic antitumor activity of oridonin and arsenic trioxide on hepatocellular carcinoma cells. Int J Oncol 40: 139-147, 2012.

28. Bu HQ, Liu DL, Wei WT, et al: Oridonin induces apoptosis in SW1990 pancreatic cancer cells via p53-and caspase-dependent induction of p38 MAPK. Oncol Rep 31: 975-982, 2014.

29. Kroemer G, Dallaporta B and Resche-Rigon M: The mitochondrial death-life regulator in apoptosis and necrosis. Annu Rev Physiol 60: 619-642, 1998.

30. McConkey DJ: Biochemical determinants of apoptosis and necrosis. Toxicol Lett 99: 157-168, 1998.

31. Strasser A, Cory S and Adams JM: Deciphering the rules of programmed cell death to improve therapy of cancer and other diseases. EMBO J 30: 3667-3683, 2011.

32. Huang YT, Huang DM, Chueh SC, et al: Alisol B acetate, a triterpene from Alismatis rhizoma, induces Bax nuclear translocation and apoptosis in human hormone-resistant prostate cancer PC-3 cells. Cancer Lett 231: 270-278, 2006.

33. Ghobrial IM, Witzig TE and Adjei AA: Targeting apoptosis pathways in cancer therapy. CA Cancer J Clin 55: 178-194, 2005.

34. Kim RH, Coates JM, Bowles TL, et al: Arginine deiminase as a novel therapy for prostate cancer induces autophagy and caspase-independent apoptosis. Cancer Res 69: 700-708, 2009. 